\title{
How pigment volume concentration (PVC) and particle connectivity affect leaching of corrosion inhibitive species from coatings
}

DOI:

10.1016/j.porgcoat.2019.05.008

\section{Document Version}

Accepted author manuscript

Link to publication record in Manchester Research Explorer

Citation for published version (APA):

Emad, S., Zhou, X., Morsch, S., Lyon, S., Liu, Y., Graham, D., \& Gibbon, S. R. (2019). How pigment volume concentration (PVC) and particle connectivity affect leaching of corrosion inhibitive species from coatings. Progress in Organic Coatings. https://doi.org/10.1016/j.porgcoat.2019.05.008

\section{Published in:}

Progress in Organic Coatings

\section{Citing this paper}

Please note that where the full-text provided on Manchester Research Explorer is the Author Accepted Manuscript or Proof version this may differ from the final Published version. If citing, it is advised that you check and use the publisher's definitive version.

\section{General rights}

Copyright and moral rights for the publications made accessible in the Research Explorer are retained by the authors and/or other copyright owners and it is a condition of accessing publications that users recognise and abide by the legal requirements associated with these rights.

\section{Takedown policy}

If you believe that this document breaches copyright please refer to the University of Manchester's Takedown Procedures [http://man.ac.uk/04Y6Bo] or contact uml.scholarlycommunications@manchester.ac.uk providing relevant details, so we can investigate your claim.

\section{OPEN ACCESS}




\title{
How pigment volume concentration (PVC) and particle connectivity affect leaching of corrosion inhibitive species from coatings
}

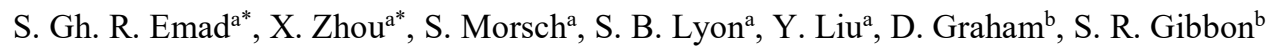 \\ a Corrosion and Protection Centre, School of Materials, The University of Manchester, The Mill, Sackville St, \\ Manchester, M13 9PL, UK \\ ${ }^{\mathrm{b}}$ AkzoNobel, Supply Chain, Research \& Development, Stoneygate Lane, Felling, Gateshead, Tyne \& Wear, NE10 0JY, \\ UK
}

\begin{abstract}
Active anti-corrosion pigments, such as strontium chromate, are essential components of many corrosion protective organic coatings, since these leach out to provide active inhibition to the metallic substrate at the defective areas of the coatings arising during service from mechanical and/or environmental damages. Currently, formulators use empirical tests to determine effective inhibitor concentration, because the factors that determine leaching behaviour are poorly understood. In this study, we present insights into leaching mechanisms by correlating the microstructure of model coatings pigmented with strontium aluminium polyphosphate hydrate (SAPH) to the transport of different species.

It is found that diffusion and transport of active species through the polymeric matrix does not significantly contribute to the leaching kinetics. Thus, leaching starts when inhibitor pigments are in direct contact with the environment via surface-breaking defects in the coating, and continues as long as the cluster of connected inhibitor pigments is in direct contact with the environment, until 3-dimensional connectivity is lost. Therefore, the extent, shape and size of the clusters of connected inhibitor pigments, as well as the solubility and dissolution rates of individual pigments, play important roles in the leaching process. Additionally, the 3-dimensional percolation threshold $\left(\mathrm{P}_{\mathrm{c}}\right)$ for pigment connectivity is proposed as a critical parameter that has significant influence on the leaching rate as well as the barrier properties of corrosion protective coatings.
\end{abstract}

Keywords: Organic Coating; Inhibitor; Pigment Volume Concentration (PVC); Percolation Threshold $\left(\mathrm{P}_{\mathrm{C}}\right)$; Inhibition; Leaching

* To whom correspondence should be addressed.

Xiaorong.Zhou@manchester.ac.uk tel: +44 (0) 1613064832

Reza.Emad@manchester.ac.uk tel: +44 (0) 1613062914 


\section{Introduction}

Organic coatings provide protection for metallic substrates by increasing the resistance of the ionic paths between the local anodes and cathodes on the surface of the metallic substrate [1-3], and by acting as a physical barrier to restrict/ prevent the transport of aggressive species to the substrate while having sufficient adhesion [4]. Nonetheless, physical ageing and interaction with environmental factors such as water/ moisture, thermal cycles, electromagnetic radiations and internal/ external mechanical stresses eventually result in formation of defects and, hence, failure of the organic coatings [5-7]. Inhibitor pigments are usually incorporated into the organic coatings to impart self-healing properties when a defect generates. Therefore, in an ideal condition, the coating acts as a reservoir for the corrosion inhibitor pigment until a defect generates and the inhibitive species leach out of the coating to interact with the substrate, which is in direct contact with the environment via the defect, to passivate the metal surface and retard the corrosion processes at the defect $[5,8-16]$.

Salts of chromate are effective corrosion inhibitors in a variety of environments and on numerous metallic substrates. In particular, strontium chromate pigments incorporated into polymeric matrices provide very efficient protection for metallic substrates at cut edges and defective areas of organic coatings. This is attributed to the sufficient solubility, release and transport of the chromate species to defective areas of the coating as well as effective inhibition of the corrosion process [11, 17-21]. Unfortunately, chromate is carcinogenic, toxic and environmentally hazardous. Therefore, its replacement with benign and environmentally friendly active inhibitors is in progress [18, 22-24]. However, despite intensive research efforts, to date, the alternative active inhibitors identified are not as effective, nor as versatile as chromate salts. This has raised an obstacle for the replacement of chromate with alternative inhibitors, particularly in demanding applications such as aerospace and coil coated strip steel [11, 24, 25].

Promising potential alternatives for chromate containing pigments include phosphate containing pigments [10, 26-29]. It is expected that these materials provide protection for the through-penetrative defects of the coatings as well as the cut edges by leaching from coatings and interacting with the metallic substrate to form effective protective layers, in comparable manner to chromate salts. However, in an analogy to the chromate containing pigments, not only should the inhibition mechanism be effective, but the leaching (solubility, release and transport) of the inhibitive species to the defective areas also needs to be sufficient in order to form the protective layer and maintain the protective film over the designated service life of the component.

Zinc phosphate was among the first generation of phosphate containing pigments. However, the low solubility of zinc phosphate is considered a reason for its relatively poor corrosion inhibition [26-28, 30-32]. Physical and chemical modifications of zinc phosphate have been performed to increase both its nominal solubility and phosphate content. This has led to the development of multiple generations of phosphatecontaining pigments such as zinc aluminium phosphate, zinc aluminium polyphosphate and strontium aluminium polyphosphate (SAPH). These generally have a higher solubility and phosphate content when 
compared with zinc phosphate [10,32-35]. This leads to the release of greater concentrations of phosphate species into the aqueous environment. However, when these pigments are incorporated into a polymeric matrix, the release and transport of the inhibitive species are affected by interaction between the polymeric binder and the pigment, as well as the microstructure of the coating layer (i.e. the presence of cavities, pores, polymer free volume and particularly the spatial distribution of different pigments and other fillers). These factors may prevent the supply of sufficient inhibitive species to suppress the corrosion process $[9,11,13$, 36-46]. Therefore, it is important to appreciate the influence of the pigment/ polymeric binder interaction and the resulting microstructure on the release and transport kinetics of the inhibitive species.

The overall aim of this research is to better understand the influence of the inhibitor pigment volume concentration (PVC) on the microstructure of coatings and the transport of different species through the organic coating, in order to identify transport paths for the release of the inhibitive species into the environment. In recent work [36], we identified the percolation threshold $\left(\mathrm{P}_{\mathrm{c}}\right)$ as a critical value for inhibitor PVC to be considered during formulation. In this study further research has been undertaken to verify the effect of the percolation threshold on the leachate concentration and the barrier properties of the coatings. Leaching rate and electrochemical impedance spectroscopy (EIS) measurements have been performed as a function of the inhibitor PVC. The effect of coating thickness on the leaching rate was also investigated. Furthermore, the microstructure of the unexposed and exposed coatings were characterised by scanning electron microscopy (SEM). Finally, energy dispersive X-ray spectroscopy (EDS) was used to investigate the influence of the inhibitor PVC on the transport of ions from the environment through the coating towards the metallic substrate.

\section{Experimental}

\subsection{Materials and specimens preparation}

Corrosion protective coatings (primer coatings) with different volume concentrations of active inhibitor pigments were formulated, and applied to flat substrates of Galfan ( $\mathrm{Zn}-5 \% \mathrm{Al})$ hot-dip coated steel, a widely used material in the coil coating industry. The polymeric binder is composed of two parts: a polyester (Dynapl LH 820-16/ 55\%) and a blocked aliphatic polyisocyanate (Tolonate D2). The inorganic pigments were strontium aluminium polyphosphate hydrate (SAPH - the active inhibitor pigment) and silica (Aerosil 200) as a rheology modifier. The SAPH pigment has an average particle size, average density and oil absorption of 2.0-3.5 $\mu \mathrm{m}, 2.9{\mathrm{~g} . \mathrm{cm}^{-3}}^{-}$and $40 \frac{\mathrm{g}}{100 \mathrm{~g}}$, respectively. It is reported [36] that SAPH comprises different components of varying chemical compositions, which may be broadly categorized into three groups: 1-strontium-rich, 2-phosphorus-rich and 3-aluminium-rich. The average of the elemental concentrations of SAPH pigments is presented in Table 1. Silica was added to all the formulations in this study at a constant volume concentration, approximately equal to $0.42 \%$, whereas coatings with different volume concentrations of SAPH pigment were prepared. The target volume concentrations of SAPH pigment in the formulated coatings were $5 \%, 10 \%, 15 \%, 20 \%, 25 \%$ and $30 \%$. In order to keep the non-volatile 
volume fractions within the liquid phase constant (approximately equal to 0.4 ), sufficient amounts of dibasic ester solvent were also added.

Table 1. An estimate of elemental concentrations of SAPH pigments. The values are averaged between five different sites. Each site is the average of the elemental compositions of $200 \mathrm{EDS}$ points obtained at $30 \mathrm{kV}$ electron beam accelerating voltage while the distances between the neighbouring EDS points were $7 \mu \mathrm{m}$.

\begin{tabular}{|c|c|c|c|c|c|c|}
\cline { 2 - 6 } \multicolumn{1}{c|}{} & $\mathrm{O}$ & $\mathrm{Sr}$ & $\mathrm{Al}$ & $\mathrm{P}$ & $\mathrm{C}$ & $\begin{array}{c}\text { Trace amounts of } \\
\mathrm{F}, \mathrm{Na}, \mathrm{Mg}, \mathrm{Si}, \mathrm{S}, \\
\mathrm{Cl}, \mathrm{Ca} \text { and } \mathrm{Zn}\end{array}$ \\
\hline $\begin{array}{c}\text { SAPH elemental } \\
\text { analysis (Weight\%) }\end{array}$ & $44.1 \pm 1.3$ & $21.1 \pm 1.5$ & $6.0 \pm 0.2$ & $18.6 \pm 0.2$ & $9.1 \pm 0.1$ & Remainder \\
\hline
\end{tabular}

Coatings were prepared by milling and comminution with a dual axes centrifuge (Speedmixer DAC150). The target weights of inorganic particles were added to the required amount of the first part of the polymeric binder in a disposable plastic cup. Zirconia beads were then added to assist in the milling and comminution process that was carried out for 5 minutes at $2400 \mathrm{rpm}$. Thereafter, the second part of the polymeric binder and the dibasic ester solvent were added, and further mixing was performed for 3 minutes at $1600 \mathrm{rpm}$. The fineness of the inorganic particles was checked by a Hegman gauge. If the fineness exceeded $10 \mu \mathrm{m}$, further milling was performed at $2400 \mathrm{rpm}$.

Table 2. The nomenclatures refer to the coated substrates in this paper, the thickness of the coatings and the target volume concentration of the SAPH pigments in the formulated coatings.

\begin{tabular}{|c|c|c|}
\hline $\begin{array}{c}\text { Nomenclature of the } \\
\text { coated substrates }\end{array}$ & $\begin{array}{c}\text { Thickness of the cured } \\
\text { coating }(\mu \mathrm{m})\end{array}$ & $\begin{array}{c}\text { Target PVC of the } \\
\text { SAPH pigments (\%) }\end{array}$ \\
\hline A-SP10 & $6.5 \pm 1.5$ & 10 \\
\hline A-SP20 & $6.8 \pm 1.0$ & 20 \\
\hline B-SP05 & $12.6 \pm 1.1$ & 5 \\
\hline B-SP10 & $12.6 \pm 1.5$ & 10 \\
\hline B-SP15 & $13.3 \pm 1.2$ & 15 \\
\hline B-SP20 & $13.0 \pm 1.3$ & 20 \\
\hline B-SP25 & $12.7 \pm 1.1$ & 30 \\
\hline B-SP30 & $12.9 \pm 1.3$ & 10 \\
\hline C-SP10 & $23.6 \pm 1.3$ & 20 \\
\hline C-SP20 & $23.0 \pm 1.2$ & \\
\hline
\end{tabular}

Wire wound K-bar applicators were used to deposit wet films of nominally $20 \mu \mathrm{m}, 40 \mu \mathrm{m}$ and $80 \mu \mathrm{m}$ thicknesses. These were applied onto standard Galfan $(95 \% \mathrm{Zn}-5 \% \mathrm{Al})$ coated steel substrates with a chrome- 
free pretreatment containing $\mathrm{Mn}, \mathrm{Ti}, \mathrm{P}, \mathrm{F}$ and $\mathrm{Si}$ at a thickness around $50 \mathrm{~nm}$. The thicknesses of Galfan layer and steel substrate were approximately $19 \pm 4 \mu \mathrm{m}$ and $430 \pm 10 \mu \mathrm{m}$ respectively. Prior to the coating application, the metal surface was degreased with acetone and dried in cool air. The coated panels were cured immediately by placing them in an air circulating oven at $300^{\circ} \mathrm{C}$ for 60 seconds. The thickness of the cured coatings was measured with scanning electron microscopy (SEM) from the cross sections of the coated substrates.

A guillotine was employed to cut the prepared panels from the coating side towards the metallic substrate to sizes suitable for electrochemical measurements $(2 \mathrm{~cm} \times 2 \mathrm{~cm})$ and leaching $(6 \mathrm{~cm} \times 7 \mathrm{~cm})$ studies. Prior to immersion in electrolyte the backs and edges of the specimens used for exposure and leaching measurements were covered with a waterproof masking tape purchased from Greentree (Verton Ltd). The backs and edges of the specimens used for the electrochemical measurements were covered with a mixture of beeswax and colophony ( $3: 1$ weight ratio) leaving $1 \mathrm{~cm}^{2}$ area of the surface of the coated substrates exposed to the electrolyte.

\subsection{Procedures and techniques}

The exposure test, EIS and leaching measurements were performed in $0.86 \mathrm{M}(5 \mathrm{wt} \%) \mathrm{NaCl}$ solution. During the measurements the beakers were kept in a water bath to maintain the temperature of the solution at $30^{\circ} \mathrm{C}$. The electrolyte was prepared using analytical reagent grade $>99.9 \%$ sodium chloride and deionized water (15 M $\Omega . c m)$. Specimens of B-SP05 and B-SP30 systems were also exposed to $0.86 \mathrm{M} \mathrm{KCl}$ solution up to 44 days for ion mobility studies. The $\mathrm{KCl}$ solution was prepared in likewise manner, i.e. with analytical reagent grade $>99.0 \%$ potassium chloride and deionized water $(15 \mathrm{M} \Omega . \mathrm{cm})$.

The specimens for inhibitor leaching were placed at the base of 2 litre beakers containing $250 \mathrm{ml}$ electrolyte. Small ( $2 \mathrm{ml})$ aliquots were removed at specific time intervals $(6,24$ hours, 3, 7, 21 and 44 days) from the start of immersion and were analysed by inductively coupled plasma atomic emission spectroscopy (ICP$\mathrm{AES}$ ) to measure the cumulative concentrations of $\mathrm{P}$ and $\mathrm{Sr}$ in the leachate solutions. The exposure specimens were also exposed in similar manner. Specimens were removed from the solution after different immersion times up to 44 days, rinsed with deionized water, dried with cool air and then kept in desiccators filled with silica gel before any further sample preparation and characterization.

The barrier properties of the coating layers were characterized with electrochemical impedance spectroscopy (EIS). These were performed in a three-electrode cell with a platinum counter electrode, a saturated calomel reference electrode (SCE) and a specimen prepared from the coated substrates as the working electrode. The measurements were performed for up to 42 days immersion using a Gamry potentiostat (reference 600) using $10 \mathrm{mV}$ perturbation potential versus the open circuit potential (OCP) over $10^{5}-10^{0} \mathrm{~Hz}$ frequency range. Three replicates of each of the coated substrates were measured. The impedance data were analysed and simulated with suitable equivalent electrical circuit using ZView 2 software. The microstructure of the unexposed and exposed coatings was characterized using scanning electron microscopy (SEM). A Ziess Ultra 55 field emission gun (FEG) SEM and a Ziess EVO50 SEM both equipped with EDS detectors were employed for 
this study. The cross sections of the coated substrates were prepared with the traditional grinding and polishing method. A detailed description is given elsewhere [36].

\section{Results and discussion}

\subsection{Microstructural characterization of the coatings}

\subsubsection{Characterization of unexposed coatings}

Scanning electron microscopy using different accelerating voltages (resulting in different analytical depths) was employed to characterize the microstructure of the unexposed and exposed coatings. Secondary electron (SE) imaging using low accelerating voltage, which is more surface sensitive, revealed protrusions on the top surfaces of coatings, and increasing the PVC results in the formation of rougher surfaces (Figure 1). This indicates that the protrusions formed due to the presence of SAPH pigment(s), which are breaking through the surface. On the other hand, SE images indicate that the polymeric matrix visible at the surface is continuous over the protrusions. However, backscattered electron (BSE) images revealed regions of different contrast on the top of, or adjacent to, the protrusions and asperities (Figure 2 (b)). These could be an indication of discontinuities and/or a thinner layer of polymeric matrix on those regions (i.e. thinner "skin layer"), both of which could increase the leaching kinetics of the species released from the inhibitor pigments. Figure 3 shows variations in surface morphology induced by an increase in the thickness of the coating. It is evident, at constant PVC $(10 \%$ or $20 \%)$, an increase in coating thickness lowers the degree of roughness on the surfaces of the coatings. Since protrusions are associated with the discontinuities on the surface, a smoother surface suggests a lower number of surface defects and/or the presence of a thicker skin layer; both of which could potentially lead to lower leaching rates from the top surface of thicker coatings.

Different SAPH pigment particles are evident from scanning electron images (Figure 4 and Figure 5). Bright particles are strontium-rich while darker ones are phosphorus- and aluminium-rich particles [36]. The SAPH pigments appear to be distributed randomly within the unexposed coatings, as illustrated in Figure 4, Figure 5 (a) and Figure 5 (d). However, increasing the SAPH PVC led to a reduction in the distances between neighbouring particles, i.e. the formation of more packed systems. Furthermore, the increase in PVC resulted in an increase in the population and size of interconnected SAPH pigment clusters. Relatively large clusters are observed when the PVC of the SAPH pigments is $\geq 20 \%$, and Figure 4 illustrates that these large clusters can in fact span the entire thickness of the coating when the SAPH PVC is $\geq 20 \%$. 

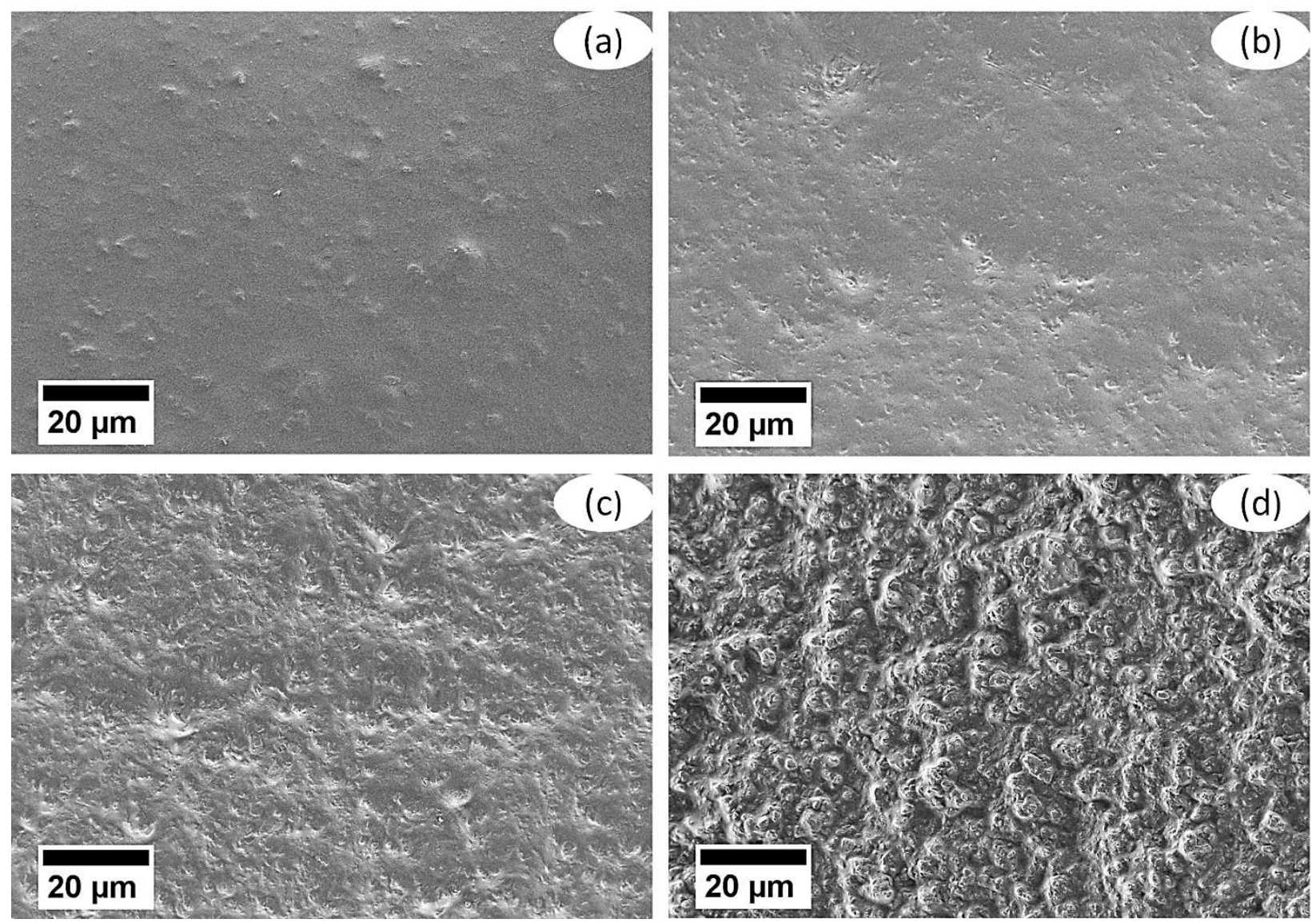

Figure 1. Secondary electron (SE) images taken at $500 \mathrm{~V}$ accelerating voltage from the top surfaces of unexposed coted metal substrates: (a) B-SP05; (b) B-SP15; (c) B-SP25 and (d) B-SP30.
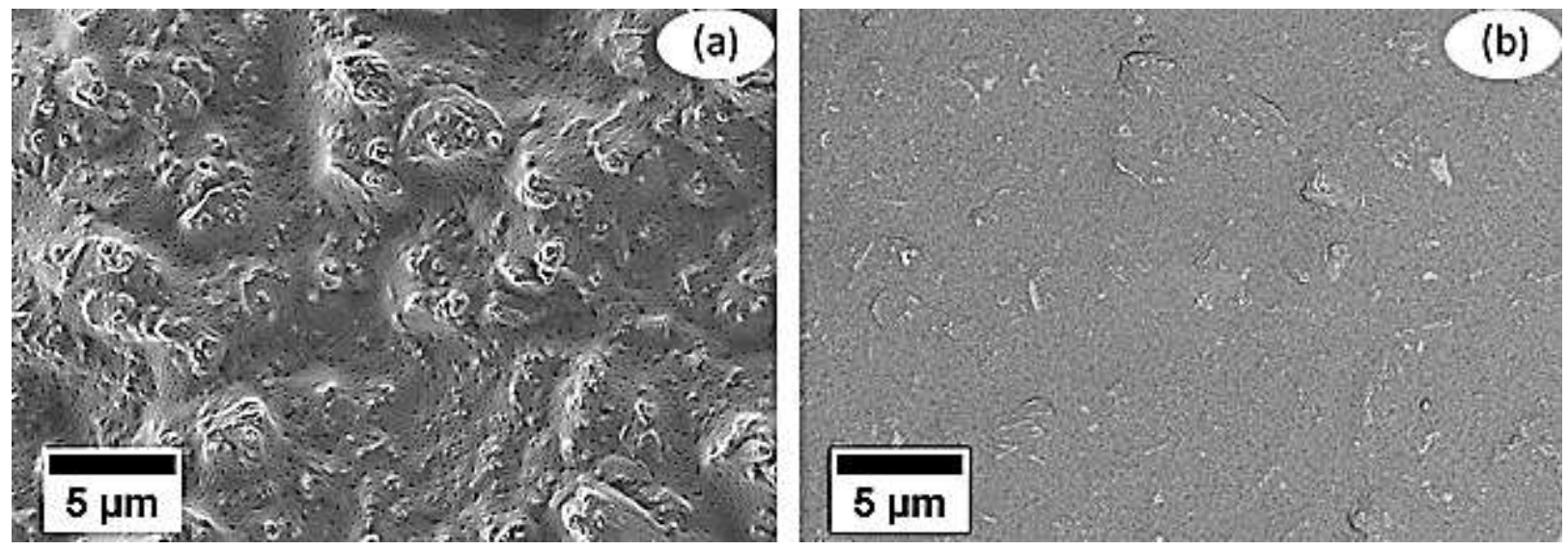

Figure 2. (a) Secondary electron and (b) backscattered electron images taken at $500 \mathrm{~V}$ accelerating voltage from the same position of the top surface of unexposed B-SP30. 


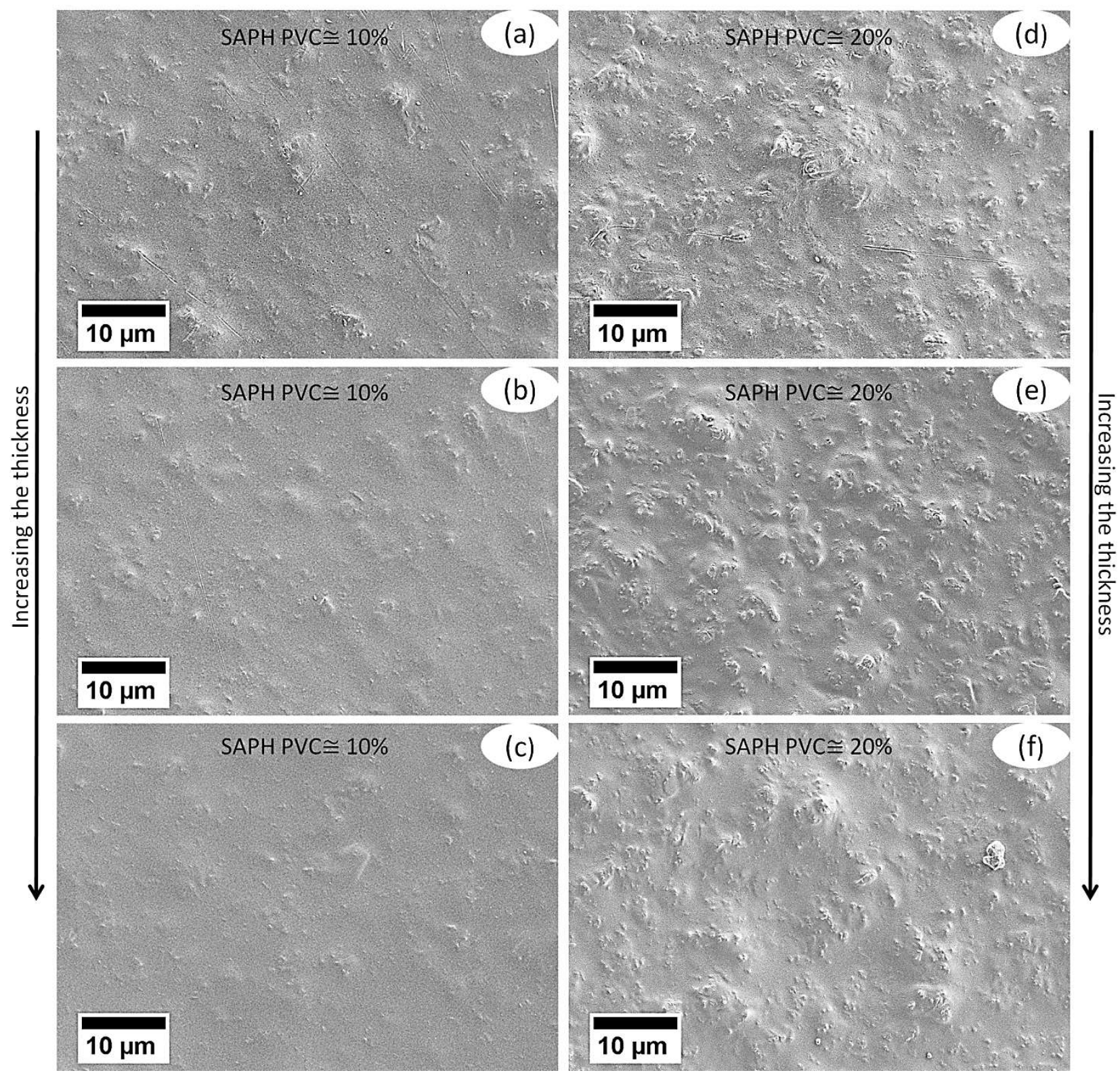

Figure 3. Secondary electron images taken at $500 \mathrm{~V}$ from the top surfaces of unexposed systems: (a) A-SP10, (b) B-SP10, (c) C-SP10, (d) A-SP20, (e) B-SP20 and (f) C-SP20. 

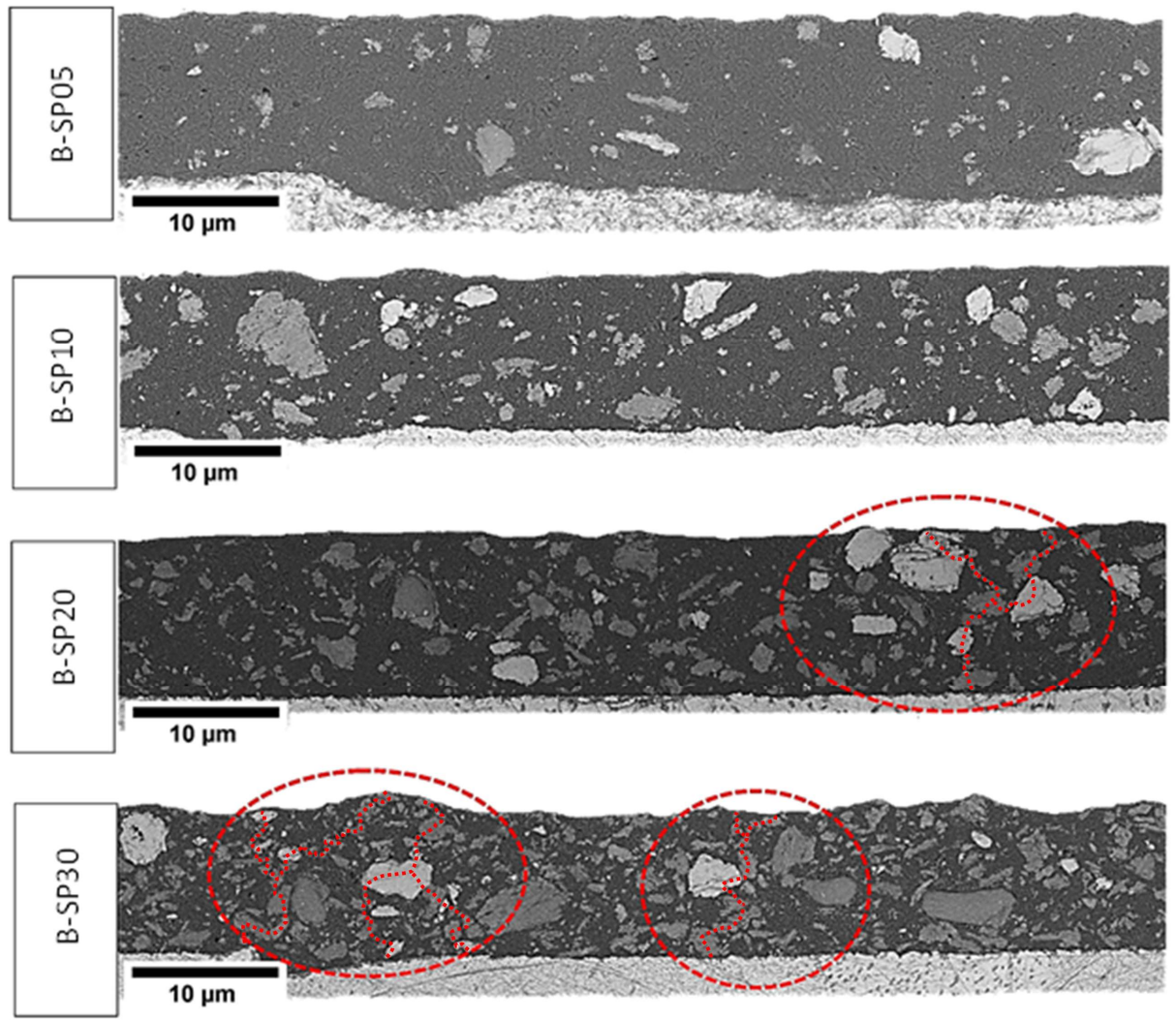

Figure 4. Backscattered electron images obtained from cross sections of unexposed coated substrates at $3 \mathrm{kV}$ accelerating voltage. The ovals indicate areas where connected SAPH clusters could be found that span the entire thickness of the coating. Dotted lines follow the connected particles.

\subsubsection{Characterization of exposed coatings}

Exposure of the coated substrates to $0.86 \mathrm{M} \mathrm{NaCl}$ solution resulted in the formation of cavity and crack-like features on the surface (Figure 6), with the majority of these appearing on the top of, or adjacent to, protrusions (corresponding to the observed discontinuities on unexposed coatings). Notably, the frequency of these features can be correlated with the initial inhibitor PVC. However, no correlation could be established between the appearance of cavity- and crack-like features on the coatings' surface and exposure time. It is therefore believed that direct contact between the environment and SAPH pigments was established at discontinuities in the coating found at or near the protrusion locations, leading to relatively rapid dissolution and removal of the SAPH particles at the loci of the discontinuities. 

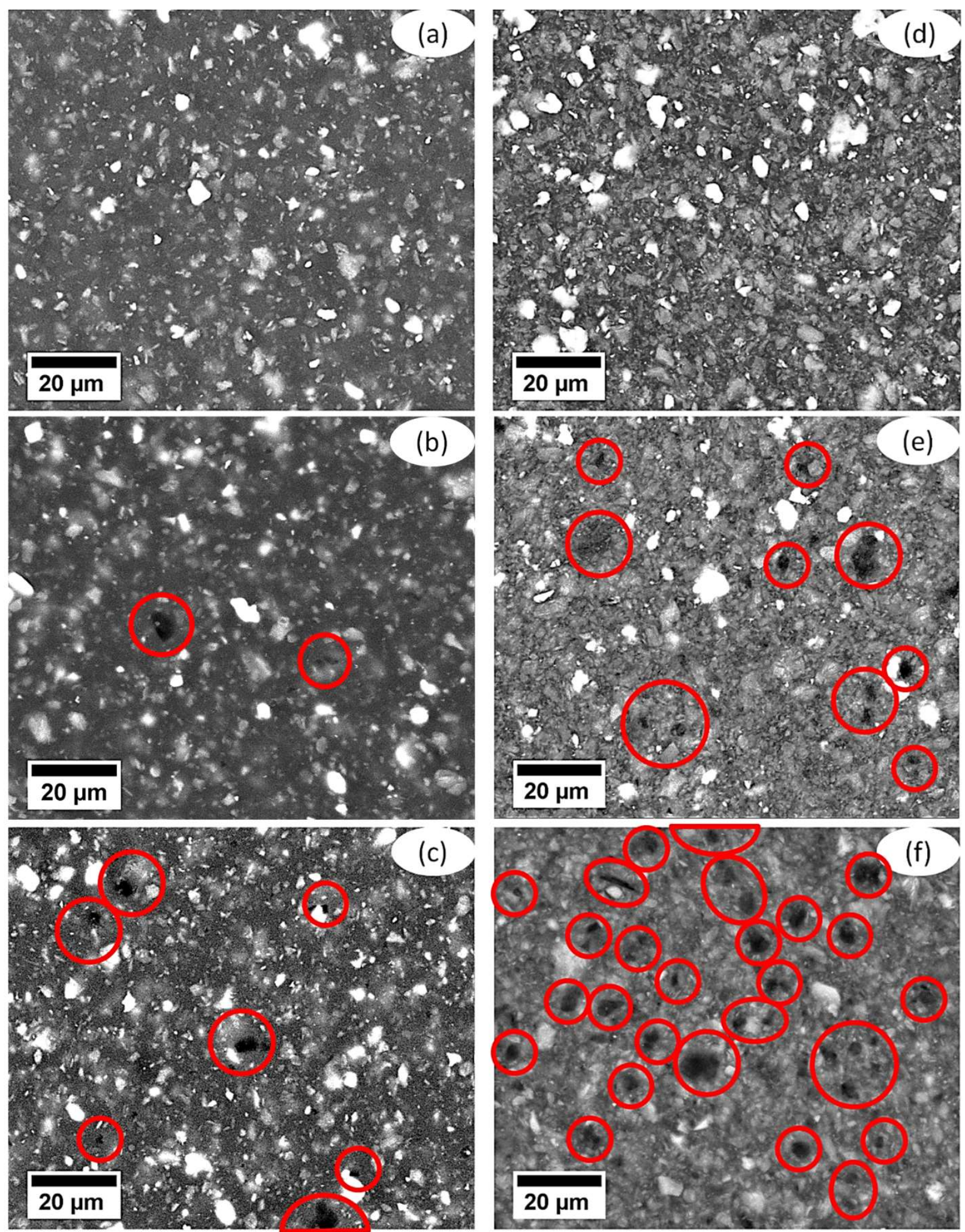

Figure 5. Backscattered electron images taken from the top surfaces of the unexposed and exposed coated substrates at $30 \mathrm{kV}$ accelerating voltage; (a) unexposed B-SP10, (b) B-SP10, which was exposed for 3 days, (c) B-SP10, which was exposed for 44 days (d) unexposed B-SP30, (e) B-SP30, which was exposed for 3 days and (f) B-SP30, which was exposed for 44 days. Ovals indicate voids and cavities that are formed after the exposure due to the dissolution and removal of SAPH pigment particles. 
A higher accelerating voltage $(30 \mathrm{kV})$ was employed to observe the microstructure of exposed specimens from increased depths of up to 2-3 $\mu \mathrm{m}$ below the surface. As is evident in Figure 5, exposure of the coated substrates to the aggressive environment resulted in the formation of voids within the coatings (i.e. the formation of a porous structure) due to the dissolution and removal of the SAPH particles [36]. Figure 5 also shows that unlike the appearance of surface cracks, the population and the frequency of these voids appear to be a function of both the initial SAPH PVC and exposure time. Therefore, it can be said that increasing the SAPH PVC results in the formation of higher number of voids within the coating. These voids can then be filled with electrolyte and provide easy transport paths for the leaching (egress) of inhibitive species as well as for the ingress of aggressive species from the environment. Nonetheless, as shown in Figure 5 (c) and (f), relatively high concentrations of SAPH pigments remain within the coatings after 44 days of immersion, and even in the case of very high PVC.
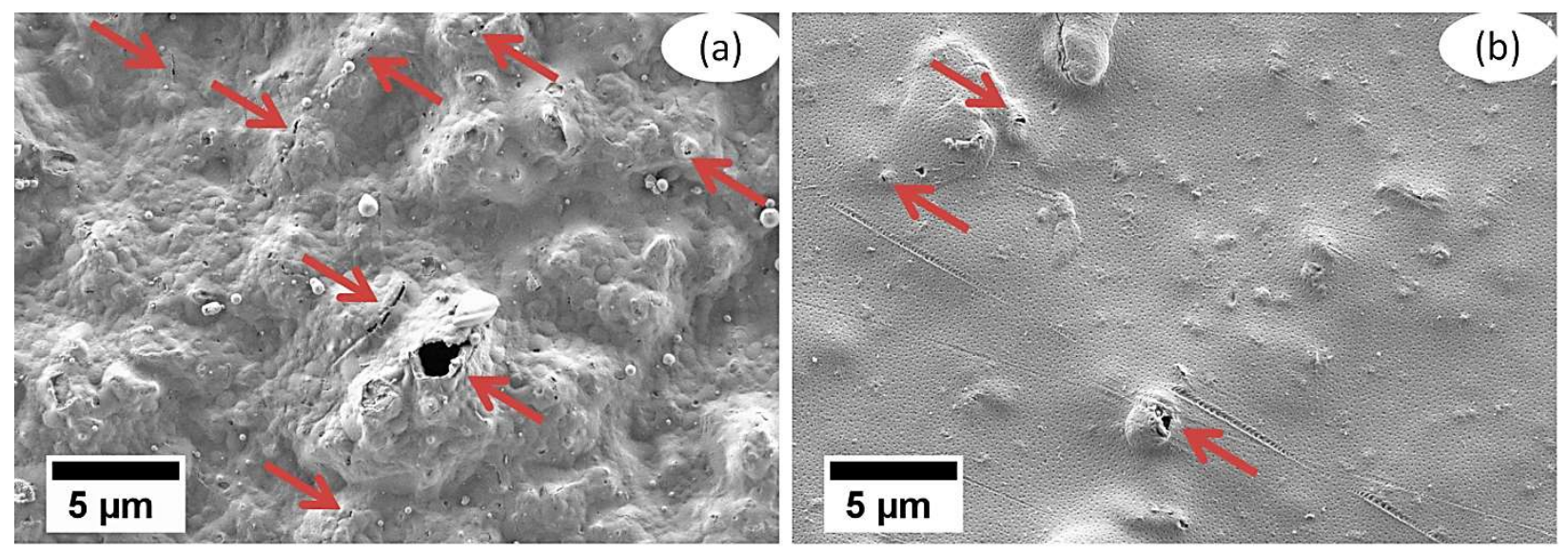

Figure 6. Secondary electron images taken at $500 \mathrm{~V}$ from the top surfaces of the coated substrates: (a) BSP30 and (b) B-SP10, which were exposed to $0.86 \mathrm{M} \mathrm{NaCl}$ for 44 days. Arrows indicate the cavity and crack-like features remaining on the surface of the coatings after exposure.

\subsection{Leaching measurements}

\subsubsection{Effect of inhibitor PVC variation}

Cumulative release profile of phosphate and strontium from the top surfaces of the coated substrates into the leachate solutions are illustrated in Figure 7. The cumulative concentrations of these species can be used as a proxy measurement of the inhibitor leaching kinetics and thus to calculate the fractions (\%) that are released into the leaching solutions (Figure 8) according to Equation 1.

Equation 1. $\quad \mathrm{F}=\frac{\mathrm{m}}{\mathrm{MPV} \rho} \times 100$

Where

F: Fraction of element (P or Sr) (\%) released per unit area of the coated substrate during exposure

$\mathrm{m}$ : Cumulative concentration of the element $(\mathrm{P}$ or $\mathrm{Sr})$ released per $\mathrm{cm}^{2}$ of the substrate 
$\rho:$ Density of SAPH pigment $=2.9 \mathrm{~g} \cdot \mathrm{cm}^{-3}$

$\mathrm{V}$ : Volume of coating $\left(\mathrm{cm}^{3}\right)$ per unit area of the substrate $\left(\mathrm{cm}^{2}\right)$

P: SAPH pigment volume concentration $(\%) \times \frac{1}{100}$

M: Weight concentration of element $(\mathrm{P} \cong 18.6 \pm 0.2 \%$ or $\mathrm{Sr} \cong 21.1 \pm 1.5 \%)$ within the SAPH pigments $(\%) \times \frac{1}{100}$

It is evident from Figure 7 and Figure 8 that strontium and phosphorus have different leaching rates. The concentrations of $\mathrm{Sr}$ released are significantly higher than the released concentration of $\mathrm{P}$ irrespective of the initial SAPH PVC. The higher release rates of Sr (compared with P) are attributed to (a) a cationic exchange processes replacing, for example, $\mathrm{Na}^{+}$or $\mathrm{K}^{+}$from the environment with $\mathrm{Sr}^{2+}$, and (b) the higher dissolution rates of the Sr-rich fraction of the SAPH conglomerate pigment particles [36].

An increase in the initial volume concentration of the SAPH pigments in the coating results in an increase in concentration of both $\mathrm{P}$ and $\mathrm{Sr}$ released. However, this is not a linear relationship since relatively higher fractions of Sr and P were leached from the coatings with higher inhibitor PVC. This indicates that leaching is not only affected by the inhibitor PVC but is also influenced by the microstructural variations within the coating. It is proposed that the formation of clusters of connected SAPH particles has a significant influence on the amount leached. As is evident in Figure 7 and Figure 8, the cumulative concentrations of $\mathrm{Sr}$ and $\mathrm{P}$ in the leachate solutions do not significantly increase after 21 days of immersion for coating containing inhibitor PVC $\leq 15 \%$. This is despite the relatively high amount of inhibitor pigment remaining within the coating after 44 days of immersion (Figure 5 (c)). On the other hand, relatively higher leach rates are observed after 21 days of immersion where the inhibitor PVC $\geq 20 \%$. In other words, the leaching process can continue at higher rates and for longer periods of time if the coating inhibitor PVC $\geq 20 \%$. As a result, the difference between the cumulative concentrations of $\mathrm{P}$ and $\mathrm{Sr}$ released from the coatings with inhibitor PVC $\leq 15 \%$ and from coatings with inhibitor PVC $\geq 20 \%$ become more noticeable with increasing immersion time (Figure 7). This phenomenon is attributed to the formation of large clusters of connected inhibitor particles within the coating where the inhibitor PVC $\geq 20 \%$.

In order to better understand the likelihood of such large cluster formation, it is informative to consider percolation theory. When two phases (e.g. phase A and phase B) are mixed to form a three dimensional continuum by a random distribution of phase B (i.e. the inhibitor) within phase A (i.e. the matrix), percolation theory suggests that the probability of a space within the three dimensional continuum (i.e. coating) being occupied with phase B depends on the volume fraction of phase B (i.e. the PVC). It is shown that at a critical probability (i.e. critical volume fraction), an extended network/cluster of phase B forms, which completely penetrates the three dimensional continuum, and has dimensions comparable to the dimensions of the system. This critical volume fraction (i.e. probability) is known as the percolation threshold $\left(\mathrm{P}_{\mathrm{c}}\right)$. The percolation threshold for random distribution of solid uniform spherical particles is 
approximately $0.16[47,48]$. However, any variation from the aforementioned ideal conditions may shift the $\mathrm{P}_{\mathrm{c}}$ to lower or higher values.

SAPH pigments are neither uniform in size nor shape [36], and this situation tends to result in an increase in the percolation threshold. The observation of extended cluster(s) of connected SAPH pigments covering the entire coating thickness at inhibitor PVC $\geq 20 \%$ (Figure 4) indicates that the $\mathrm{P}_{\mathrm{c}}$ for the SAPH pigments is likely to be slightly lower than this value. Therefore, it is presumed that the $\mathrm{P}_{\mathrm{c}}$ for this system is between 0.16-0.20, and when the inhibitor PVC $\geq 20 \%$, large clusters of connected SAPH particles span the coating thickness. Hence, upon dissolution and removal of a single particle belonging to a connected cluster, a cavity forms that is connected to neighbouring particle. These cavities are open to the external environment and, hence, become filled with electrolyte, facilitating the dissolution and release of inhibitive species from the next connected neighbouring particle. In addition the electrolyte-filled cavities provide easy transport paths for released species. This process continues until the large cluster of connected SAPH particles becomes exhausted of inhibitive species. In contrast, when the inhibitor PVC $\leq 15 \%$, clusters of connected SAPH pigments are not large enough to span the coating thickness and the regions between smaller clusters are filled with the polymeric binder. The polymeric barrier limits the dissolution, release and transport of the inhibitive species from the neighbouring clusters of the SAPH particles and, hence, lowers the leaching kinetics and leachate concentrations.
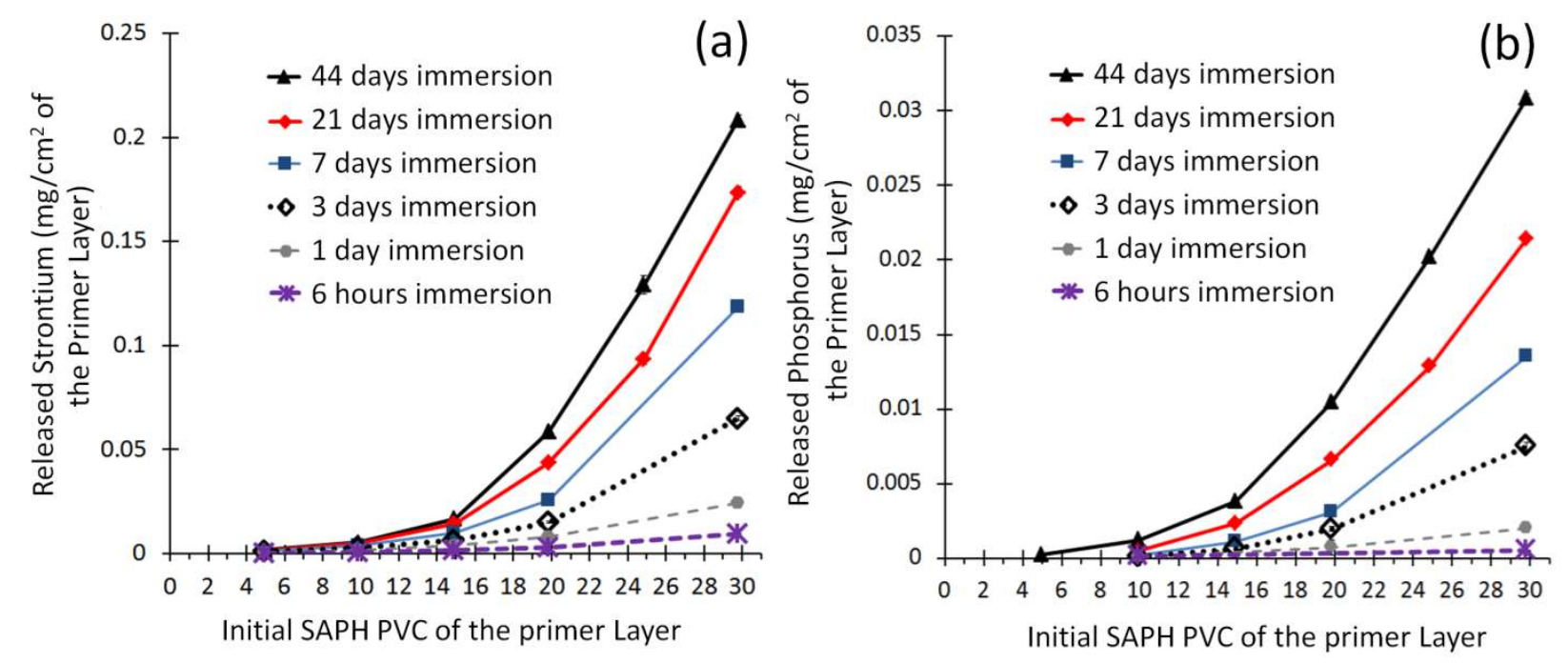

Figure 7. Cumulative release of (a) strontium and (b) phosphorus per unit area of the coated substrates (BSPxx set) as a function of initial SAPH pigment volume concentration for different periods of time. The error bars are less than the marker size (not all the aliquots removed from the leachate solution of B-SP25 were sent for ICP-AES analysis. Only the samples removed after 21 and 44 days were analysed). 


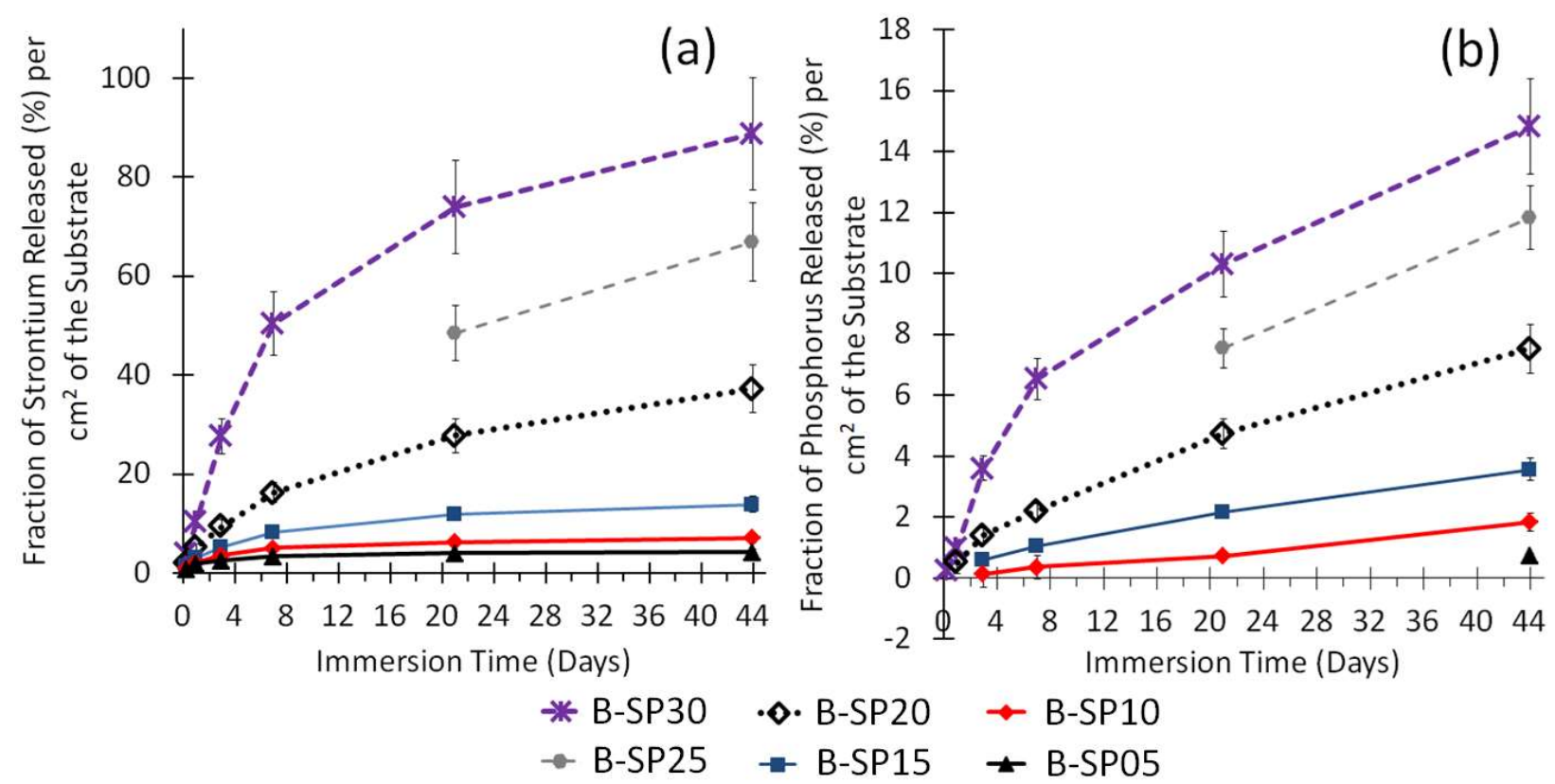

Figure 8. Fractions (\%) of: (a) strontium and (b) phosphorous that released per $\mathrm{cm}^{2}$ of the coated substrates of B-SPxx set (not all the aliquots removed from the leachate solution of B-SP25 were sent for ICP-AES analysis. Only the samples removed after 21 and 44 days were analysed).

\subsubsection{Effect of thickness variation}

Figure 9 shows the effect of thickness variation on the cumulative concentrations of strontium in the leachate solutions. Note that the trends for phosphorus (not shown) were comparable at lower concentrations. It is evident that irrespective of the initial concentrations of the SAPH pigments, thinner coatings released greater amounts of strontium during the first few days after the start of immersion. This is attributed to a higher number of surface defects and discontinuities on the surface of the thinner coatings, which allow direct contact of the inhibitor pigment with the environment. Moreover, this observation confirms the influence of direct contact of the inhibitor pigments with the environment on the kinetics of the early stages of the leaching process, although it is, of course, also dependent on the solubility and release rate(s) of the inhibitive species. Thus, in this case $\mathrm{P}$ and $\mathrm{Sr}$ showed similar leaching kinetics but at different rates.

On the other hand, after prolonged immersion times, different behaviours are observed depending on the initial volume concentration of the SAPH pigments. Thus, at $10 \% \mathrm{PVC}$, the cumulative concentration of strontium released from a thinner coating was greater than that released from a thicker coating over the entire period of immersion (44 days), Figure 9. This might appear counter-intuitive since the mass of the SAPH pigments per $\mathrm{cm}^{2}$ of the surface of the thicker coating is approximately double that in the thinner coating. However, at an inhibitor PVC of $10 \%$, large clusters of connected SAPH pigments that span the coating thickness are not yet formed. Therefore, any species released from the inhibitor pigments not in direct contact with the environment via surface defects, needs to be transported through the polymeric binder phase in order to leach out. Therefore, it is suggested that release and transport through the polymeric binder is the limiting factor that has halted the release of $\mathrm{Sr}$ from the coatings with 10\% SAPH PVC at prolonged immersion times. Moreover, a large number of surface-breaking particles within the thinner coating has led to the release of higher initial concentrations of Sr into the leachate solution from the start of immersion. 
This suggests that incorporation of inhibitor pigments within the polymeric binder does not by itself provide a readily available reservoir for the leaching of the inhibitive species but that direct contacts between inhibitor pigments and with the external environment is necessary for leaching process. If clusters of connected inhibitor pigments are effectively encapsulated with a defect-free layer of the polymeric binder, then the leaching process is severely restricted.
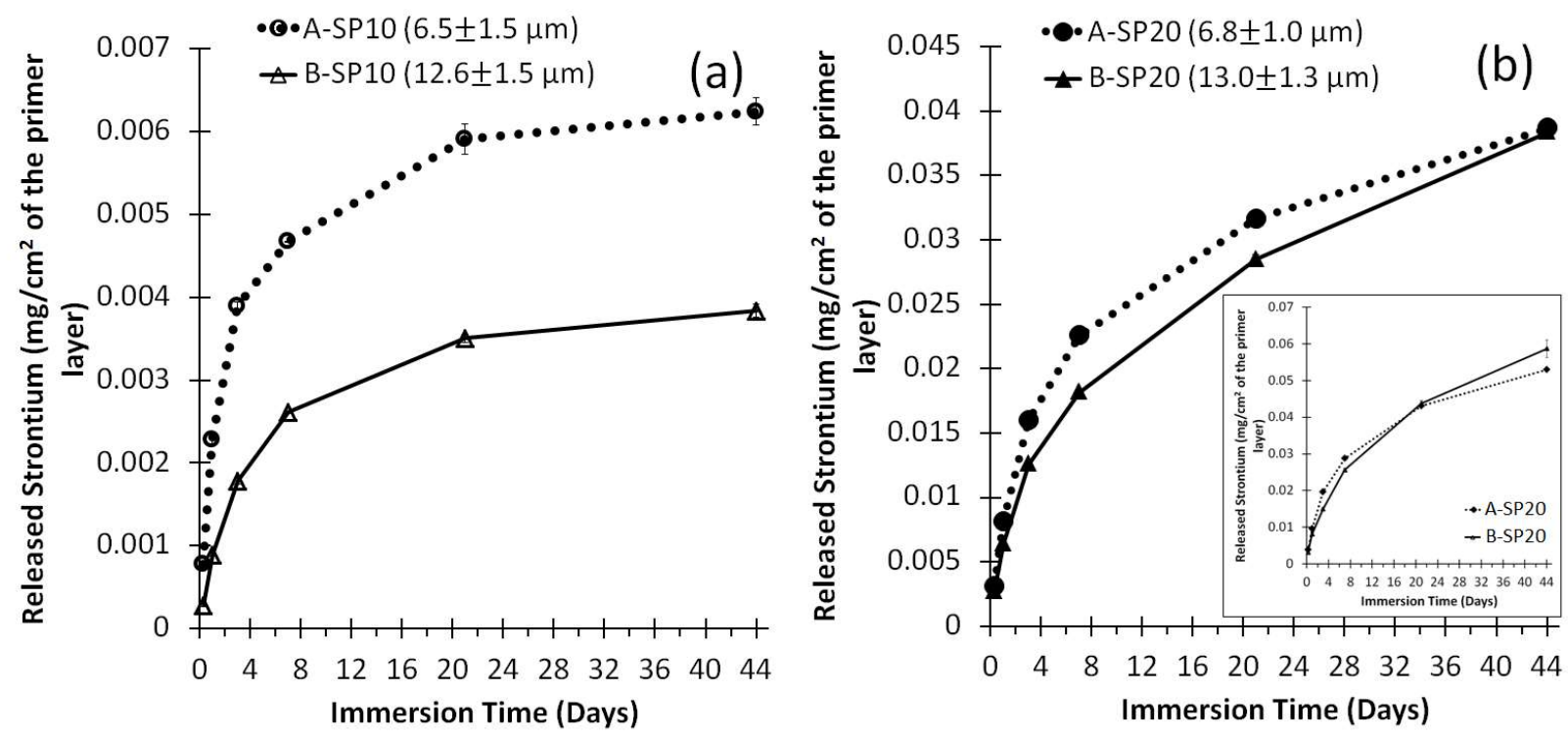

Figure 9. Comparison of the cumulative concentrations of Sr released from coatings of different thicknesses but similar volume concentrations of SAPH pigments; (a) SAPH PVC $\cong 10 \%$ and (b) SAPH PVC $\cong 20 \%$. The inset of (b) shows the results for the repeat of measurements for coatings with $20 \%$ SAPH PVC.

In contrast, the cumulative concentrations of strontium released from coatings with $20 \%$ volume concentration of the SAPH pigments do not plateau after 44 days of immersion, although the leaching rates gradually reduce with time. After 44 days of immersion, the cumulative concentrations of strontium released from the thinner and the thicker coatings are comparable to each other. This is attributed to the formation of large clusters of connected SAPH particles for the systems with inhibitor PVC $\geq 20 \%$ that span the entire coating thickness. As discussed in the previous section, when the inhibitor PVC $\geq 20 \%$, large clusters of connected SAPH particles exist within the coating. Therefore, thicker coating can provide larger reservoirs of leachable materials via connected clusters without being restricted by transport in the polymeric matrix. For thinner coating the readily available reservoir is smaller and gets exhausted and depleted of inhibitor more rapidly. This confirms that when the inhibitor PVC is above the percolation threshold, large clusters of connected active inhibitor pigments with a critical size form within the coating layer.

The described leaching measurements from the coating top surface as functions of thickness and inhibitor PVC have enabled us to develop a general model for leaching processes that is also applicable to the leaching from cross-sections (i.e. leaching from through-thickness penetrative defects and leaching from cut edges). In this model diffusion through the polymeric binder is negligible. The organic coating mainly acts as a reservoir for inhibitor pigments and acts as a barrier to impede the corrosion process. Leaching can only start when the inhibitor particles are in direct contact with the environment via defects and discontinuities 
that allow dissolution and release of the inhibitive species, which may result in the formation of passive layer on the metal surface at the defect. When an inhibitor particle is in direct contact with the environment via a defect/ discontinuity, and it belongs to a cluster of connected inhibitor particles, the dissolution and removal of that particle results in the formation of a cavity. This cavity will also be in direct contact with neighbouring particles. Therefore, the cavity forms a passage that facilitates the dissolution and transport of the inhibitive species from the neighbouring particle into the leachate solution, and leaching can continue until the cluster becomes depleted. Therefore, in the early stages of leaching, all species are released from those pigments that are in direct contact with the environment, whereas after prolonged exposure inhibitive species will only release and leach out from inhibitor particles that are connected to the networks of connected voids and cavities formed due to the removal of inhibitor pigments. Figure 10 shows a simplified schematic diagram illustrating the leaching model for coatings with two different volume concentrations of inhibitor pigments; above and below the percolation threshold. In the case of a coating with inhibitor PVC $\geq$ $\mathrm{P}_{\mathrm{c}}$ the leaching process can continue even after " $\mathrm{t}_{4}$ " since a cluster of connected inhibitor pigments has not yet been completely depleted of inhibitor pigments. However, the leaching kinetics at these stages depend on the transport phenomena in the complex structure of connected voids and cavities. On the other hand, the leaching stops at " $t_{4}$ " for coatings with inhibitor $\mathrm{PVC}<\mathrm{P}_{\mathrm{c}}$ since the clusters of connected inhibitor pigments which are in direct contact with the environment become depleted of inhibitor pigments at an earlier stage. Of course, leaching may re-commence if additional defects are generated due to degradation of the polymeric matrix, or due to further service damage that directly connects individual particles or clusters of connected particles to the environment. 


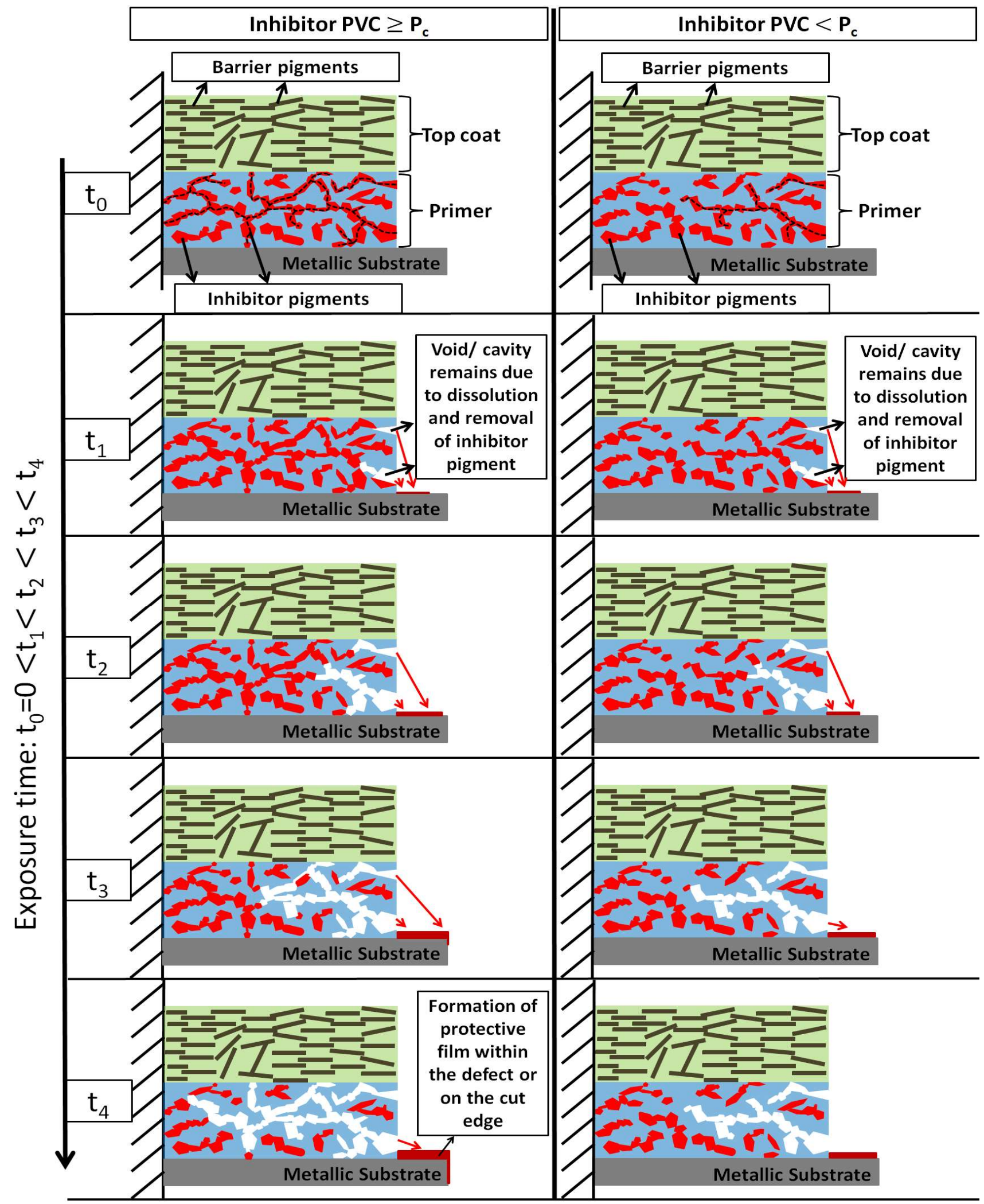

Figure 10. Simplified schematic diagram illustrating the leaching process as a function of time for two coatings with two different inhibitor pigment volume concentrations. Left column: inhibitor $\mathrm{PVC} \geq \mathrm{P}_{\mathrm{c}}$. Right column: inhibitor PVC $<\mathrm{P}_{\mathrm{c}}$. This simplified diagram is meant to visualize the leaching process/ model only, and the protective film formation is just an illustration of what may happen. Also, the interactions at the interface of coating/ metallic substrate, variation of the local environment, the degradation of organic coating and formation of corrosion products over the exposure period and their influence on leaching process are not considered. The black dashed lines at $t_{0}$ follow the clusters of connected inhibitor pigments which are in direct contact with the environment. Red arrows indicate leaching of inhibitive species into the defective areas. 


\subsection{EIS measurements}

The barrier properties of the B-SP05, B-SP10, B-SP15, B-SP20 and B-SP30 coating systems were measured using electrochemical impedance spectroscopy. In all cases only one time-constant was evident over the frequency range used to obtain the EIS data during the first few days of immersion. However, as time progressed a second time-constant became evident. The first time-constant at high frequencies can be attributed to the impedance of the coating layer. Equivalent electrical circuits with constant phase elements (CPE) are used to fit the data and quantify the barrier properties (resistance) of the coatings. Equivalent electrical circuits used for fitting of the EIS measurements, Bode plots of EIS measurements and the fitted spectra to the EIS measurements are available in electronic supplementary information.

In general, the resistances of the coatings drop significantly during the first few days from the start of immersion, which indicates uptake of the environment and the gradual development of defects in the coatings. Beyond this time a more gradual decrease in resistance is observed that depends on the initial PVC of the SAPH pigments. The resistance decrease in coatings above the percolation threshold (B-SP20 and BSP30) is greater than those of the B-SP05, B-SP10 and B-SP15 systems where the PVC is less than the $\mathrm{P}_{c}$. Indeed, the resistance of the B-SP05, B-SP10 and B-SP15 systems remain comparable to each other up to the end of measurements with a slight increase as function of the inhibitor PVC (i.e. B-SP05 < B-SP10 < BSP15), and are approximately an order of magnitude higher than the resistance of B-SP20, Figure 11.

As is illustrated in Figure 11, after 14 days of immersion and up to the end of measurement, the magnitude of coatings resistances were: B-SP30 « B-SP20 « B-SP05 < B-SP10 < B-SP15. Thus, in coatings with inhibitor PVC greater than the percolation threshold, a substantial reduction in coating resistance is evident after 14 days, whereas for coatings with inhibitor PVC less than the percolation threshold the coating resistance remains relatively higher for longer.

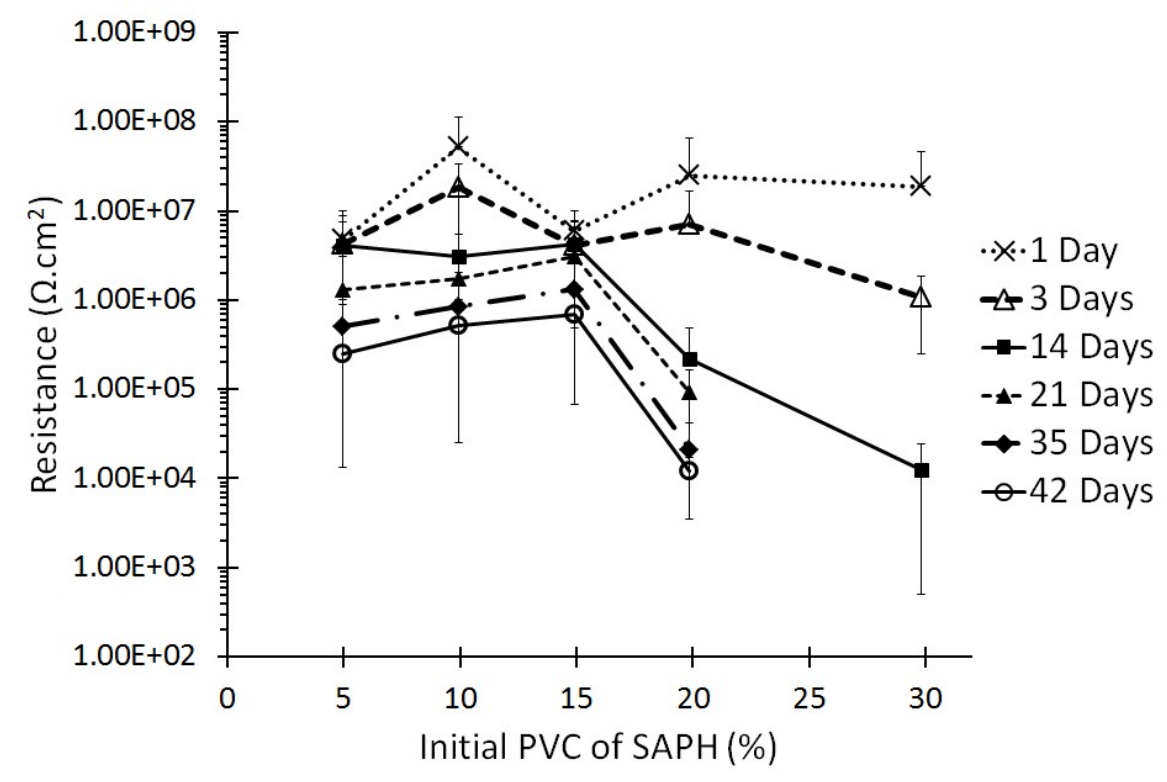


Figure 11. Variations of the resistances of the coatings of B-SPxx set as a function of the initial SAHP PVC for different periods of time. The resistances for each coating system are averaged between three independent specimens measured at approximately similar times after the start of immersions.

The time dependence of the coating resistance correlates with the leachate concentrations and the fractions of materials released from the coatings. The fractions of materials released from B-SP05, B-SP10 and B-SP15 are not significantly different from each other, whereas the fractions of materials released from B-SP20 and B-SP30 are significantly higher. We attribute this to the formation of spanning networks and clusters of connected SAPH pigments within the coatings with SAPH PVC $\geq 20 \%$. The variations of the coating resistances can be justified in a similar manner. The dissolution, release and leaching of the materials into the environment result in the formation of voids and cavities within the coatings that can be filled with electrolyte, and act as the transport paths for charge carrier species through the thickness of the coatings. Therefore, when networks of connected SAPH pigment exist within the coating, networks of connected voids and cavities are an inevitable consequence providing easy conductive paths from the environment to the metallic substrate.

The data presented indicate that an increase in the inhibitor PVC varies the transport properties through the bulk of coatings, which becomes more noticeable when the inhibitor PVC is above the $\mathrm{P}_{\mathrm{c}}$. On the other hand, as long as the inhibitor PVC is lower than the $\mathrm{P}_{c}$, the barrier properties of the coatings are comparable and the transport of aggressive species such as $\mathrm{Cl}^{-}$and $\mathrm{Na}^{+}$from the environment towards the metallic substrate are likely to be similarly restricted.

\subsection{Potassium transport through the coatings}

Specimens of B-SP05 and B-SP30 systems were immersed in $0.86 \mathrm{M} \mathrm{KCl}$ solution to investigate the transport of potassium ions as a function of inhibitor PVC. The characteristic X-ray line of potassium $\mathrm{K}_{\alpha}$ obtained from the (apparently) intact regions of the polymeric binder of B-SP05 coating that was immersed for 44 days are barely above the noise level of the background of the EDS spectra (e.g. spectra 5 and 6 of Figure 12). This indicates low concentrations of potassium have penetrated these regions of the polymeric binder (i.e. transport of potassium through the binder itself is negligible).

As discussed in section 3.2.1, the SAPH pigments have cationic exchange properties therefore substitution of $\mathrm{Sr}^{2+}$ with $\mathrm{K}^{+}$will result in an increase in the concentration of $\mathrm{K}^{+}$within the pigment particles. For the B-SP05 system that was exposed for 44 days, the increase in potassium concentration is evident only for those particles that are just below the surface (i.e. just below the coating/environment interface), e.g. spectra 1 and 2 of Figure 12, whereas the concentration of potassium within the particles that are further away from the surface is not in the detectable range of EDS (e.g. spectra 3 and 4 of Figure 12). This confirms that there is negligible transport of $\mathrm{K}^{+}$through the polymeric matrix. In contrast, relatively high concentrations of potassium were detected through the entire thickness of the B-SP30 coating after only 21 days of immersion, Figure 13 (a). This indicates that potassium ions can be transported via facile conductive paths formed by extended networks of connected SAPH pigments. 

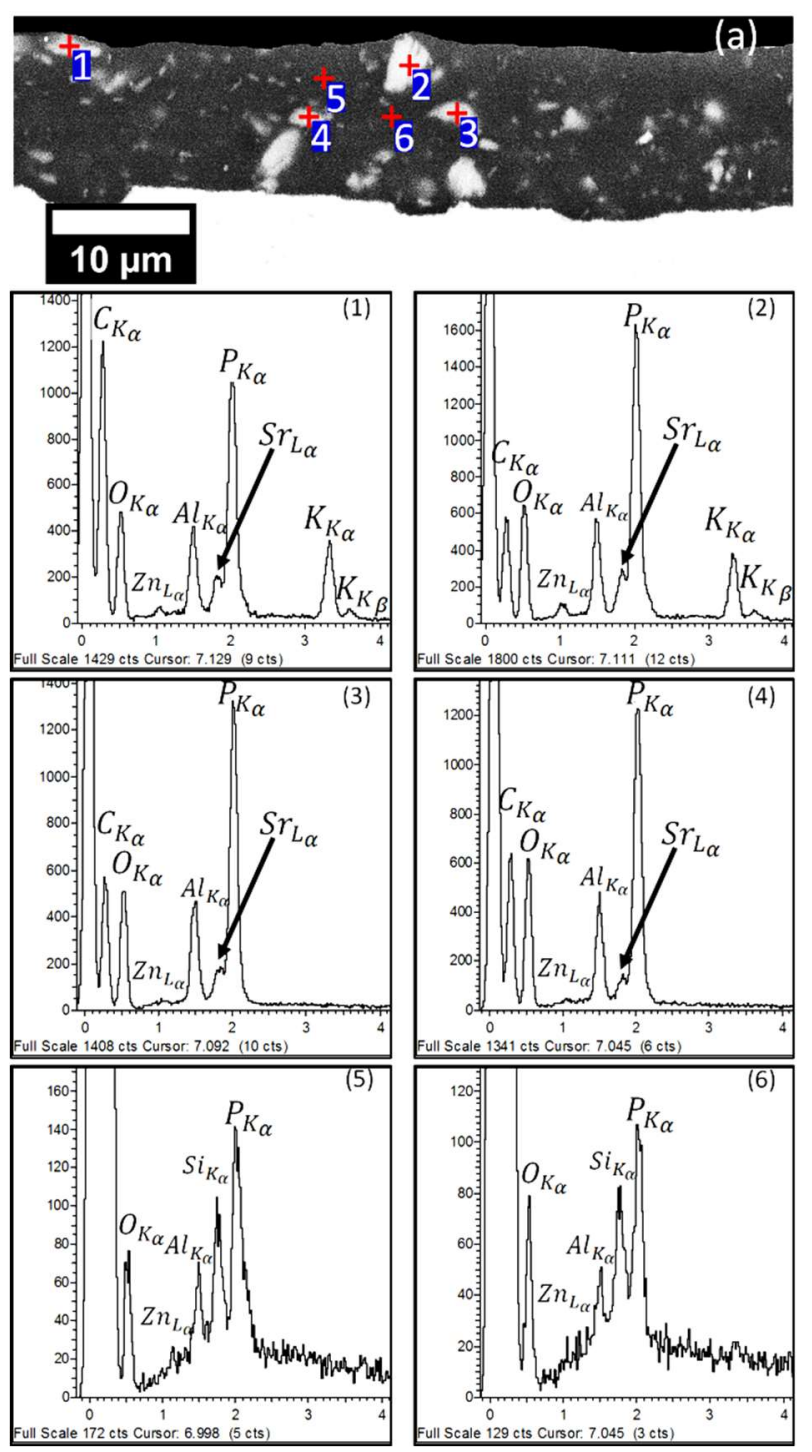

Figure 12. (a) BSE image obtained at $20 \mathrm{kV}$ accelerating voltage from the cross section of B-SP05 system exposed for 44 days in $0.86 \mathrm{M} \mathrm{KCl}$ solution. (1), (2), (3), (4), (5) and (6) are the EDS spectra obtained from $1,2,3,4,5$ and 6 EDS points, respectively, indicated by the crosses on image (a).

The EDS data is consistent with the EIS and leaching measurements, and shows that increasing the SAPH PVC not only introduces defects and discontinuities on the surface but also varies the transport properties through the bulk of the coatings. These variations increase the leaching rates, the leachate concentrations and enhance the ingress of aggressive species from the environment towards the metallic substrate, especially when the inhibitor PVC is above the percolation threshold. 


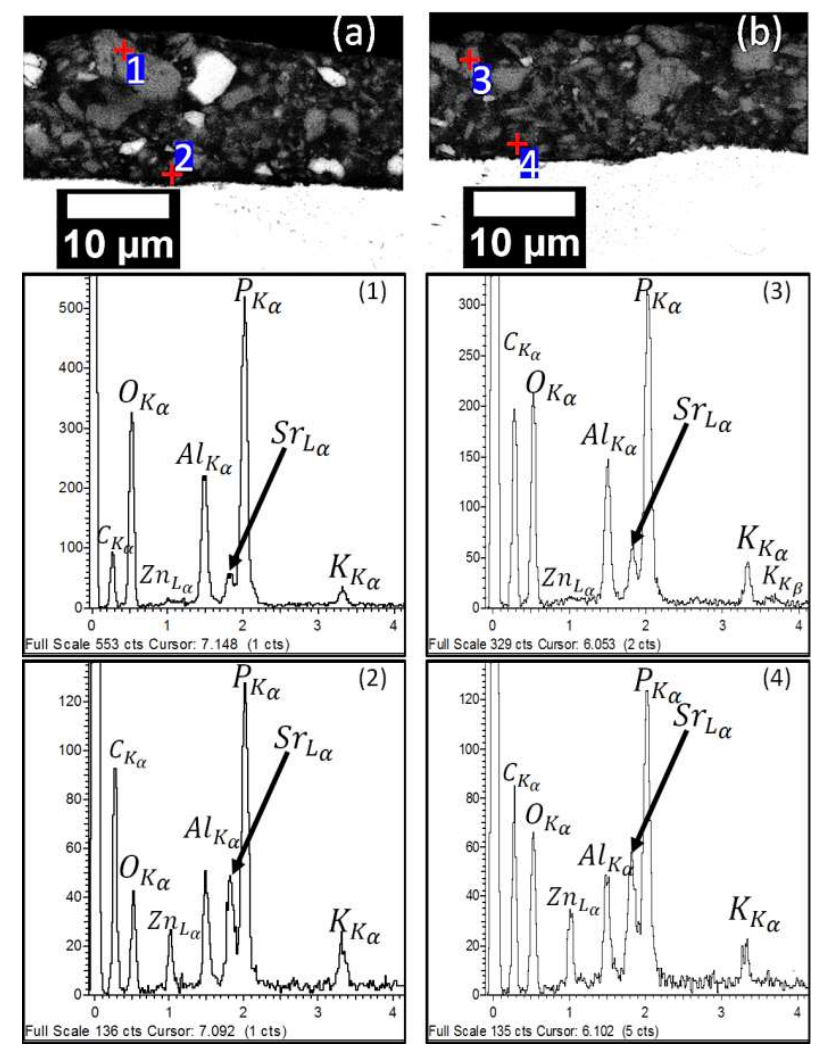

Figure 13. (a) and (b) BSE images obtained at $20 \mathrm{kV}$ accelerating voltage from the cross sections of B-SP30 system exposed in $0.86 \mathrm{M} \mathrm{KCl}$ solution for 21 and 44 days respectively. (1), (2), (3) and (4) are the EDS spectra obtained from 1, 2, 3 and 4 EDS points, respectively, indicated by the crosses on images.

In summary, the evidence presented in this report suggests that when inhibitor pigments are encapsulated within a defect-free, intact polymeric matrix, significant leaching does not take place and, basically, organic coating acts as a reservoir for inhibitive species. As a corollary, leaching only takes place when a defect/ discontinuity exists that allows direct contact between the environment and inhibitor pigments. When leaching starts from a cluster of connected inhibitor pigments, it will continue until the cluster becomes exhausted of these species. The removal of the connected inhibitor particles results in the formation of networks of connected voids that act as easy transport paths which facilitate the dissolution, release and transport of further inhibitive species from inhibitor particles now connected directly via these voids to the environment. As suggested by Hughes et al. [38], the early stages of the leaching process are controlled by the dissolution and solubility rates of the inhibitor pigments that are in direct contact with environment, whereas the leaching process over long exposure time is controlled by the transport of inhibitive species through the networks of voids and cavities which remain after dissolution of inhibitor pigments.

A continuous supply of the inhibitor over the service life of the component, required for long life, is highly dependent on the dissolution, release and transport rates of the species released from the inhibitor pigments. For instance, in B-SP30, which is above the percolation threshold, and clusters of SAPH pigments exist that span the coating thickness allowing a continuous supply of strontium and phosphorus into the environment. However, after 44 days of immersion approximately $89 \%$ of $\mathrm{Sr}$ is released into the environment while only $15 \%$ of the phosphorus content of the coating layer is released. Thus, coatings with higher PVC may become 
depleted earlier than expected and, hence, may not be able to supply inhibitive species over the intended service lifetime of the coating. Therefore, the PVC of the inhibitor pigments should be selected with respect to the inhibitor efficiency, dissolution, release and transport rates as well as the percolation threshold. When an inhibitor pigment has high solubility and release rates, the volume concentration of the inhibitor pigments should not be significantly above the percolation threshold to avoid fast depletion of the primer layer from that species. On the other hand, when the inhibitor has low solubility and release rates, having the concentration of the inhibitor pigments significantly above the percolation threshold results in higher leachate concentrations and provides the required transport path for the leaching of higher concentrations of inhibitive species, whereas the low solubility and release rates prevent the fast depletion of the primer from the inhibitive species. However, as EIS and EDS measurements reveal, when the inhibitor PVC is above the percolation threshold it allows higher ingress of the aggressive species into the primer layer as well. This indicates that an inhibitor PVC, which corresponds to an inhibitor cluster size, should be selected that balances the leaching of inhibitive species with the ingress of the aggressive species in a way that minimizes the detrimental effect of the ingress of the aggressive species whilst supplying sufficient concentration of inhibitive species for the formation and maintenance of the protective film over the designed service life of the component.

\section{Conclusions}

The coatings microstructure, the leaching behaviour and the barrier properties have been investigated as functions of inhibitor PVC and the thickness of the coatings in order to establish the relationship between the microstructure and the leaching mechanisms, and to identify the dominant transport paths for the leaching of inhibitive species.

An increase in inhibitor PVC leads to a proportionately higher increase in the leaching rate, which results in faster depletion of the inhibitor pigments from the coatings with higher inhibitor PVC. This is attributed to the difference in spatial distribution of the inhibitor pigments since the increase in inhibitor PVC leads to the formation of clusters of connected inhibitor pigments. Consequently, the percolation threshold $\left(\mathrm{P}_{c}\right)$ is considered a critical value for inhibitor pigment volume concentration which significantly influences the total leaching at prolonged exposure times. In order for the leaching process to start, the inhibitor pigments should be in direct contact with the environment via defects and discontinuities in the polymeric matrix (i.e. "skin layer"). If the inhibitors are encapsulated with a continuous and defect-free layer of polymeric binder, then leaching rate is negligible. The dominant transport paths for the leaching of inhibitive species are through the voids and cavities formed initially or left behind after the dissolution of inhibitor pigments. Based on the leaching data and microstructural characterization, a model for the leaching of inhibitive species has been proposed.

Furthermore, EIS measurements corroborate this model, by showing that the barrier properties of coatings increase with the increase in the inhibitor PVC as long as inhibitor PVC is kept below the $\mathrm{P}_{\mathrm{c}}$. In contrast, when inhibitor PVC is above the $\mathrm{P}_{\mathrm{c}}$, a significant reduction of the barrier properties is observed, which is 
attributed to the formation of large clusters of inhibitor pigments that span the entire coating thickness. These spanning cluster form low resistance pathways due to the dissolution of inhibitor pigments, which allow egress and ingress of species through the coating. This is consistent with the observation that transport of $\mathrm{K}^{+}$ from the environment through the polymeric binder is negligible, whereas relatively high concentrations of $\mathrm{K}^{+}$are detected adjacent to the metallic substrates when the large spanning clusters of inhibitor pigments are present within the coating.

\section{Acknowledgments}

The Authors are grateful to AkzoNobel for financial support and materials.

\section{Data availability}

The raw/processed data required to reproduce these findings cannot be shared at this time as the data also forms part of an ongoing study.

\section{References}

[1] J.E.O. Mayne, The mechanism of the protective action of an unpigmented film of polystyrene, Journal of Oil Colour Chemistry Association, 32 (1949) 481-487.

[2] J.E.O. Mayne, HOW PAINTS PREVENT CORROSION, Anti-Corrosion Methods and Materials, 1 (1954) 286290.

[3] J.E.O. Mayne, The mechanism of the protection of iron and steel by paint, Anti-Corrosion Methods and Materials, 20 (1973) 3-8.

[4] W. Funke, H. Haagen, Empirical or Scientific Approach to Evaluate the Corrosion Protective Performance of Organic Coatings, Industrial \& Engineering Chemistry Product Research and Development, 17 (1978) 5053.

[5] S.B. Lyon, R. Bingham, D.J. Mills, Advances in corrosion protection by organic coatings: What we know and what we would like to know, Progress in Organic Coatings, 102, Part A (2017) 2-7.

[6] G.P. Bierwagen, D.E. Tallman, Choice and measurement of crucial aircraft coatings system properties, Progress in Organic Coatings, 41 (2001) 201-216.

[7] D.Y. Perera, Effect of pigmentation on organic coating characteristics, Progress in Organic Coatings, 50 (2004) 247-262.

[8] A.E. Hughes, I.S. Cole, T.H. Muster, R.J. Varley, Designing green, self-healing coatings for metal protection, NPG Asia Mater, 2 (2010) 143-151.

[9] S.A. Furman, F.H. Scholes, A.E. Hughes, D. Lau, Chromate leaching from inhibited primers: Part II: Modelling of leaching, Progress in Organic Coatings, 56 (2006) 33-38.

[10] R. Naderi, S.Y. Arman, S. Fouladvand, Investigation on the inhibition synergism of new generations of phosphate-based anticorrosion pigments, Dyes and Pigments, 105 (2014) 23-33.

[11] R.L. Howard, I.M. Zin, J.D. Scantlebury, S.B. Lyon, Inhibition of cut edge corrosion of coil-coated architectural cladding, Progress in Organic Coatings, 37 (1999) 83-90.

[12] I.M. Zin, S.B. Lyon, V.I. Pokhmurskii, M.C. Simmonds, Inhibition of steel and galvanised steel corrosion by zinc and calcium ions in the presence of phosphate, Corrosion Engineering, Science and Technology, 39 (2004) 167-173.

[13] S.A. Furman, F.H. Scholes, A.E. Hughes, D.N. Jamieson, C.M. Macrae, A.M. Glenn, Corrosion in artificial defects. II. Chromate reactions, Corrosion Sci., 48 (2006) 1827-1847. 
[14] A. Hughes, S. Furman, T. Harvey, S. Hardin, P. Corrigan, F. Scholes, T. Muster, P. White, H. Fischer, Selfhealing coating systems for corrosion protection, in: Proceedings of the First International Conference on Self Healing Materials, 18-20, Noordwijk aan Zee, The Netherlands, Wiley-VCH, 2007.

[15] P. Visser, M. Meeusen, Y. Gonzalez-Garcia, H. Terryn, J.M.C. Mol, Electrochemical Evaluation of Corrosion Inhibiting Layers Formed in a Defect from Lithium-Leaching Organic Coatings, J. Electrochem. Soc., 164 (2017) C396-C406.

[16] P. Visser, Y. Liu, H. Terryn, J.M.C. Mol, Lithium salts as leachable corrosion inhibitors and potential replacement for hexavalent chromium in organic coatings for the protection of aluminum alloys, Journal of Coatings Technology and Research, 13 (2016) 557-566.

[17] E.W. Brooman, Modifying organic coatings to provide corrosion resistance-Part III: Organic additives and conducting polymers, Metal Finishing, 100 (2002) 104-110.

[18] E.W. Brooman, Modifying organic coatings to provide corrosion resistance-Part I: Background and general principles, Metal Finishing, 100 (2002) 48-53.

[19] L. Fedrizzi, F. Deflorian, G. Boni, P.L. Bonora, E. Pasini, EIS study of environmentally friendly coil coating performances, Progress in Organic Coatings, 29 (1996) 89-96.

[20] N. Granizo, J.M. Vega, D. de la Fuente, B. Chico, M. Morcillo, lon-exchange pigments in primer paints for anticorrosive protection of steel in atmospheric service: Anion-exchange pigments, Progress in Organic Coatings, 76 (2013) 411-424.

[21] O.O. Knudsen, A. Forsgren, Corrosion Control Through Organic Coatings, 2017 ed., CRC Press, United States, 2017.

[22] E.W. Brooman, Modifying organic coatings to provide corrosion resistance: Part II-Inorganic additives and inhibitors, Metal Finishing, 100 (2002) 42-53.

[23] R.L. Twite, G.P. Bierwagen, Review of alternatives to chromate for corrosion protection of aluminum aerospace alloys, Progress in Organic Coatings, 33 (1998) 91-100.

[24] J. Sinko, Challenges of chromate inhibitor pigments replacement in organic coatings, Progress in Organic Coatings, 42 (2001) 267-282.

[25] P. de Lima-Neto, A.P. de Araújo, W.S. Araújo, A.N. Correia, Study of the anticorrosive behaviour of epoxy binders containing non-toxic inorganic corrosion inhibitor pigments, Progress in Organic Coatings, 62 (2008) 344-350.

[26] A. Amirudin, C. Barreau, R. Hellouin, D. Thierry, Evaluation of anti-corrosive pigments by pigment extract studies, atmospheric exposure and electrochemical impedance spectroscopy, Progress in Organic Coatings, 25 (1995) 339-355.

[27] F. Askari, E. Ghasemi, B. Ramezanzadeh, M. Mahdavian, The corrosion inhibitive properties of various kinds of potassium zinc phosphate pigments: Solution phase and coating phase studies, Progress in Organic Coatings, 85 (2015) 109-122.

[28] F. Deflorian, I. Felhosi, Electrochemical Impedance Study of Environmentally Friendly Pigments in Organic Coatings, Corrosion, 59 (2003) 112-120.

[29] I.M. Zin, S.B. Lyon, V.I. Pokhmurskii, Corrosion control of galvanized steel using a phosphate/calcium ion inhibitor mixture, Corrosion Sci., 45 (2003) 777-788.

[30] P. Kalenda, A. Kalendová, D. Veselý, Properties of anticorrosion pigments depending on their chemical composition and PVC value, Pigment \& Resin Technology, 35 (2006) 188-199.

[31] B. del Amo, R. Romagnoli, V.F. Vetere, L.S. Hernández, Study of the anticorrosive properties of zinc phosphate in vinyl paints, Progress in Organic Coatings, 33 (1998) 28-35.

[32] M.R. Heydarpour, A. Zarrabi, M.M. Attar, B. Ramezanzadeh, Studying the corrosion protection properties of an epoxy coating containing different mixtures of strontium aluminum polyphosphate (SAPP) and zinc aluminum phosphate (ZPA) pigments, Progress in Organic Coatings, 77 (2014) 160-167.

[33] R. Naderi, M.M. Attar, Electrochemical assessing corrosion inhibiting effects of zinc aluminum polyphosphate (ZAPP) as a modified zinc phosphate pigment, Electrochim. Acta, 53 (2008) 5692-5696.

[34] R. Naderi, M.M. Attar, Application of the electrochemical noise method to evaluate the effectiveness of modification of zinc phosphate anticorrosion pigment, Corrosion Sci., 51 (2009) 1671-1674.

[35] R. Naderi, M.M. Attar, The inhibitive performance of polyphosphate-based anticorrosion pigments using electrochemical techniques, Dyes and Pigments, 80 (2009) 349-354.

[36] S.G.R. Emad, X. Zhou, S.B. Lyon, G.E. Thompson, Y. Liu, G. Smyth, D. Graham, D. Francis, S.R. Gibbon, Influence of volume concentration of active inhibitor on microstructure and leaching behaviour of a model primer, Progress in Organic Coatings, 102, Part A (2017) 71-81. 
[37] A.E. Hughes, A. Trinchi, F.F. Chen, Y.S. Yang, I.S. Cole, S. Sellaiyan, J. Carr, P.D. Lee, G.E. Thompson, T.Q. Xiao, Revelation of Intertwining Organic and Inorganic Fractal Structures in Polymer Coatings, Advanced Materials, 26 (2014) 4504-4508.

[38] A.E. Hughes, A. Trinchi, F.F. Chen, Y.S. Yang, I.S. Cole, S. Sellaiyan, J. Carr, P.D. Lee, G.E. Thompson, T.Q. Xiao, The application of multiscale quasi 4D CT to the study of SrCrO4 distributions and the development of porous networks in epoxy-based primer coatings, Progress in Organic Coatings, 77 (2014) 1946-1956.

[39] A. Nazarov, D. Thierry, T. Prosek, N. Le Bozec, Protective Action of Vanadate at Defected Areas of Organic Coatings on Zinc, J. Electrochem. Soc., 152 (2005) B220-B227.

[40] T. Prosek, D. Thierry, A model for the release of chromate from organic coatings, Progress in Organic Coatings, 49 (2004) 209-217.

[41] J. Mardel, S.J. Garcia, P.A. Corrigan, T. Markley, A.E. Hughes, T.H. Muster, D. Lau, T.G. Harvey, A.M. Glenn, P.A. White, S.G. Hardin, C. Luo, X. Zhou, G.E. Thompson, J.M.C. Mol, The characterisation and performance of $\mathrm{Ce}(\mathrm{dbp}) 3$-inhibited epoxy coatings, Progress in Organic Coatings, 70 (2011) 91-101.

[42] F.H. Scholes, S.A. Furman, A.E. Hughes, T. Nikpour, N. Wright, P.R. Curtis, C.M. Macrae, S. Intem, A.J. Hill, Chromate leaching from inhibited primers: Part I. Characterisation of leaching, Progress in Organic Coatings, 56 (2006) 23-32.

[43] T. Prosek, D. Thierry, Mobility and Mode of Inhibition of Chromate at Defected Areas of Organic Coatings Under Atmospheric Conditions, Corrosion, 60 (2004) 1122-1133.

[44] A.E. Hughes, A. Trinchi, F.F. Chen, Y.S. Yang, S. Sellaiyan, J. Carr, P.D. Lee, G.E. Thompson, T.Q. Xiao, Structure and Transport in Coatings from Multiscale Computed Tomography of Coatings-New Perspectives for Eelectrochemical Impedance Spectroscopy Modeling?, Electrochim. Acta, 202 (2016) 243-252.

[45] M. van Soestbergen, V. Baukh, S.J.F. Erich, H.P. Huinink, O.C.G. Adan, Release of cerium dibutylphosphate corrosion inhibitors from highly filled epoxy coating systems, Progress in Organic Coatings, 77 (2014) 1562-1568.

[46] S. Sellaiyan, A.E. Hughes, S.V. Smith, A. Uedono, J. Sullivan, S. Buckman, Leaching properties of chromate-containing epoxy films using radiotracers, PALS and SEM, Progress in Organic Coatings, 77 (2014) 257-267.

[47] R. Zallen, The physics of amorphous solids, Wiley, New York, 1983.

[48] H. Scher, R. Zallen, Critical Density in Percolation Processes, The Journal of Chemical Physics, 53 (1970) 3759-3761. 